Special Reviews

Juntendo Medical Journal

2014. 60 (4), 293-299

\title{
A Paradigm Shift for the Concept of Diabetic Nephropathy
}

\author{
TOMOHITO GOHDA*, YASUHIKO TOMINO*
}

*Division of Nephrology, Department of Internal Medicine, Juntendo University Faculty of Medicine, Tokyo Japan

Diabetic nephropathy (DN) is increasing rapidly worldwide. In Japan, over the past two decades, the proportion of $\mathrm{DN}$ in patients with new-onset hemodialysis induction dramatically increased along with an exponential increase in the number of diabetic patients. Regarding the pathophysiology of DN, it was previously thought to be very simple, i.e. the development/progression of $\mathrm{DN}$ was able to be explained only in terms of hyperglycemia, genetic factors, and so on. However, it has become evident that many factors, such as advanced glycation end-products (AGEs), oxidative stress, and chronic inflammation, are involved in the pathogenesis of DN. With respect to progression process of DN, we thought it to be very difficult to halt the progression of this disease if the urinary excretion of albumin increased up to the level of macroalbuminuria (an albuminuria of more than $300 \mathrm{mg}$ / $\mathrm{g} \cdot \mathrm{Cr}$ ), a stage which has been called "the point of no return". Now even diabetic patients with a nephrotic range of proteinuria sometimes regress to levels of normo- or microalbuminuria with the benefit of advances in diabetic treatment.

This review article describes some of the knowledge that has been gained from our previous studies and also reviews advances in clinical and basic research in DN based on published literature over the past decades.

Key words: diabetic nephropathy, inflammation, biomarker, $\mathrm{KK}-\mathrm{A}^{\mathrm{y}}$ mouse

\section{Current situation of diabetic nephropathy from the point of view of epidemiology}

9.5 million people in Japan have diabetes mellitus, and the number with diabetes has rapidly increased by appropriate $40-$ fold in six decades ${ }^{12}{ }^{2}$. According to the 2012 annual report by the Japanese Society of Dialysis Therapy (JSDT), the total number of dialysis patients was 304, 592, and since 1998 the leading cause of end stage renal disease (ESRD) has become diabetes $(44.1 \%)$ instead of chronic glomerulonephritis (19.1\%) (Figure-1) ${ }^{3)}$. Although the dialysis population added had increased by appropriately 10 thousand patients annually in the past, the number of recent new-onset dialysis patients has been reduced by almost half. Moreover, the annual proportion of the annual dialysis population attributed to diabetes has almost stopped increasing in the past 5 years.

Two landmark clinical studies, the Diabetes

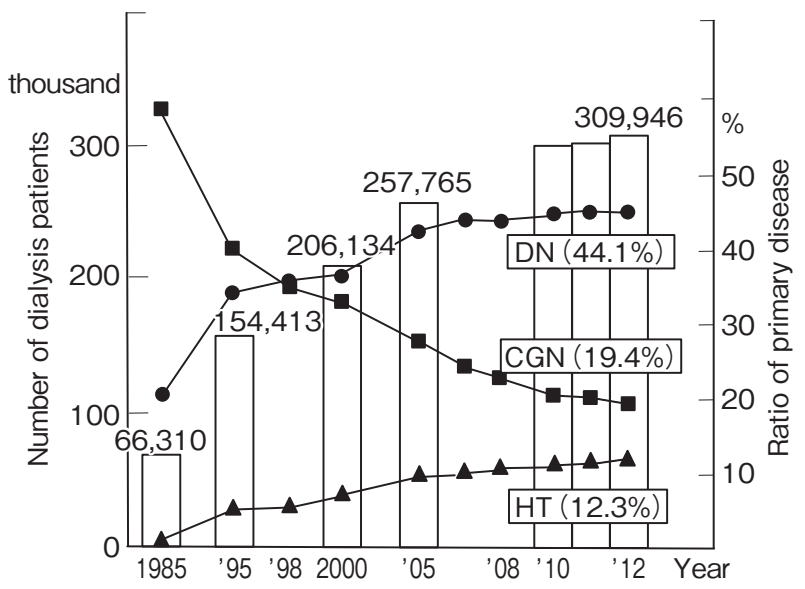

Figure-1 The number of hemodialysis patients and cause of ESRD

The number of hemodialysis patients has been increasing over the past several decades, but new-onset dialysis cases have declined somewhat in recent years. As of 1998, the leading cause of ESRD is now diabetes (44.1\%) instead of chronic glomerulonephritis (19.1 \%). Adapted from the Japanese Society for Dialysis Therapy (JSDT) annual report 2012 with permission of the JSDT.

Corresponding author: Tomohito Gohda

Division of Nephrology, Department of Internal Medicine, Juntendo University Faculty of Medicine

2-1-1 Hongo, Bunkyo-ku, Tokyo 113-8421, Japan

TEL/FAX: +81-3-5802-1065 E-mail: goda@juntendo.ac.jp

333rd Triannual Meeting of the Juntendo Medical Society "Medical Research Update” 〔Held on May 17, 2014〕

〔Received June 5, 2014〕 
Control and Complications Trial (DCCT ${ }^{4)}$ and the Collaborative study ${ }^{5}$, performed two decades ago in patients with type 1 diabetes (T1D), demonstrate a good prognosis for an individual with diabetes over time. In the DCCT, intensive blood glucose control by insulin prevents or slows development/progression in patients with early diabetic nephropathy (DN) better than those with conventional therapy and poor glycemic control. In the Collaborative study, the angiotensin converting enzyme (ACE) inhibitor captopril protected against declining renal function and increasing albuminuria, and this effect is likely independent of any change in blood pressure. Similarly, the effect of another type of renin-angiotensin-aldosterone system (RAS) inhibitor, angiotensin II type 1 receptor blocker (ARB), in the prevention of progression of $\mathrm{DN}$ in patients with type 2 diabetes (T2D) was confirmed in subsequent a work of the Reduction of Endpoints in NIDDM with the Angiotensin II Antagonist Losartan (RENAAL) study ${ }^{6}$. Now RAS inhibitors are an established first line therapy, not only to lower blood pressure, but also to decrease albuminuria, leading to renal and cardiovascular protective effects.

Over the past 20 years, significant progress in the development of modern medicines for diabetes and its associated diseases has made treatment easy. Given this situation, there is the potential for the decreased prevalence of DN. Yet, the prevalence of kidney disease in patients with diabetes remains unchanged according to the cross-sectional analyses of the Third National Health and Nutrition Examination Survey (NHANES III) from 19881994 and NHANES from 1999-2008 in the United States ${ }^{7)}$. In detail, the prevalence of albuminuria is decreasing, but the prevalence of glomerular filtration rate (GFR) loss is rather increasing among patients with diabetes. Thus, it can't be denied that the core of DN might be shifting from albuminuria to impaired GFR.

Taken together, the decrease in new-onset dialysis cases might be only temporary due to a postponement in reaching ESRD. In fact, the cumulative risk of ESRD remained high in a cohort of patients attending the Joslin Clinic in Boston and in nationwide cohort of patients in Finland, referred to as the FinnDiane cohort, over nearly 2 decades. Therefore, new treatment strategies that further slow the progression of GFR loss are required since the residual risk in patients who receive this optimal care such as insulin and RAS inhibitors remains extremely high.

\section{Progression process of nephropathy from the point of view of observational studies}

In the classical model of DN (Figure-2A), once urinary excretion levels of albumin increase and reach macroalbuminuria levels, it was previously thought that there would never be a regression to normo- and microalbuminuria, with the GFR beginning to decline, and then eventually reaching to ESRD. This progression process was considered to be one-way.

Pancreas transplantation is a unique therapy that is able to keep long-lasting euglycemia without exposing the patients to the risks of severe hypoglycemia. Islet cell transplantation is also able

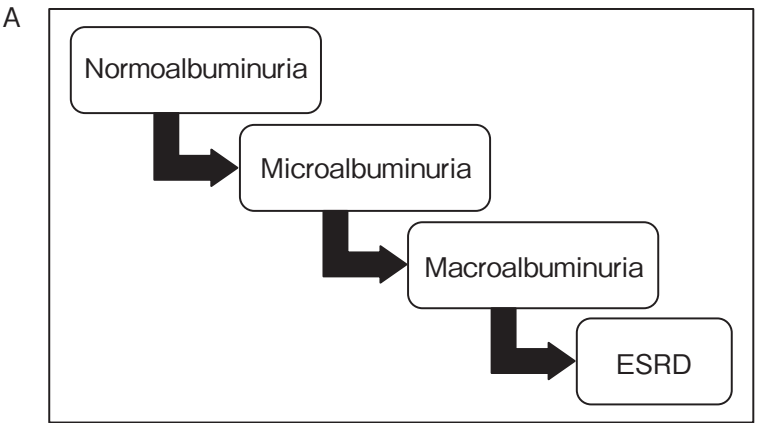

B

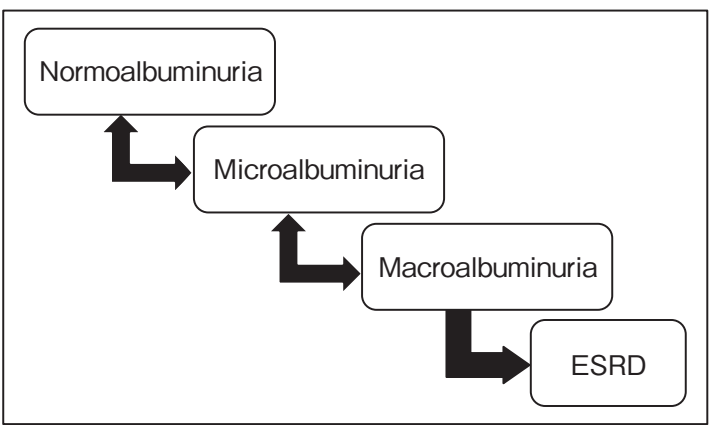

Figure-2 Progression pattern of diabetic nephropathy A. Classic model of diabetic nephropathy

Once urinary excretion levels of albumin increase and reach macroalbuminuria, the progression process was considered to be one-way.

B. New model of diabetic nephropathy

Even in patients with macroalbuminuria or nephrotic syndrome, some of them can regress to micro- or normoalbuminuria. However, once progressive renal decline has occurred, DN progresses relentlessly toward ESRD. 
to achieve an insulin independence condition, but it is difficult to keep euglycemia for a long time of period, in spite of significant advances in isolation techniques and postoperative management. Fioretto $e t a l{ }^{8)}$ examined histologic renal findings before and after pancreas transplantation in patients with T1D who had mild to advanced diabetic glomerular lesions at the time of transplantation. Glomerular lesions were not significantly changed at 5 years after pancreas transplantation. Meanwhile, diabetic glomerular lesions, such as Kimmelstein-Wilson nodules, were dramatically reversed at 10 years after pancreas transplantation, suggesting that DN is not an irreversible renal disease, the considerable amount of time to ameliorate the histological improvement notwithstanding. We should also note that this treatment is not approved for recipients with low pretransplant GFR because nephrotoxic immunosuppressive drugs, such as cyclosporine and tacrolimus, are required over a lifetime to prevent acute and chronic alloimmunue host responses after transplantation. Nonetheless, these results have encouraged many nephrologists and endocrinologists directly involved in the treatment of DN as they learned for the first time this disease is likely to be reversible. After this report, many researchers have looked at the natural history of $\mathrm{DN}$ in greater detail. The results have shown that microalbuminuria is more likely to regress to normoalbuminuria than to progress to macroalbuminuria ${ }^{9)}$. Even in patients with macroalbuminuria or nephrotic syndrome, some have experienced a complete remission (Figure-2B) ${ }^{10)-12)}$. Concerning renal function, once the progressive renal decline (a GFR loss of at least $3.3 \%$ per year) has occurred, the progression process is one-way. This is observed in $10 \%$ of type 1 diabetic patients with normoalbuminuria, $25 \%$ of those with microalbuminuria, and 50 $\%$ of those with macroalbuminuria ${ }^{13)}{ }^{14}$. These results have opened the door to a new model of DN.

\section{Circulating soluble TNF receptors and novel biomarker to predict renal function decline}

Albuminuria is one of the first asymptomatic clinical manifestations of microvascular injury in diabetes. An increase in albuminuria was thought to change in accordance with a decrease in the GFR. However, the established formula to estimate the
GFR from age, sex, and serum creatinine levels revealed that the abnormal urinary excretion of albumin and impaired GFR do not always exacerbate in parallel with each other, and are rather two separable manifestations of $\mathrm{DN}^{15}$. Currently, albuminuria lacks both specificity and sensitivity as a prognostic biomarker for progressive $\mathrm{DN}$, as $\mathrm{DN}$ is sometimes able to progress without an increase in albuminuria and even in the presence of normal albuminuria ${ }^{16)}{ }^{17)}$. Also, a current large-scale study, the BP-lowering arm of the Action in Diabetes and Vascular disease: preterAx and diamicroN-MR Controlled Evaluation (ADVANCE), demonstrated that patients with microalbuminuria and a decrease in GFR, but with microalbuminuria and no decrease in GFR, have poor renal outcomes ${ }^{18)}$. However, most clinical studies done thus far have used only albuminuria as a substitute clinical (surrogate) endpoint for the progression of DN because it takes a long time for patients with an early stage of DN to reach ESRD. Thus, prior treatment of patients with early DN might not be enough for preventing GFR loss, although it has achieved great success in terms of the reduction of albuminuria. Therefore, reliable diagnostic biomarkers that identify individuals at high risk for GFR loss or ultimately ESRD, other than albuminuria, should be required.

Among many different candidate biomarkers, the Joslin group found that serum concentrations of soluble tumor necrosis factor receptor 1 (TNFR1) and 2 (TNFR2) as possible biomarkers for predicting GFR loss in patients with a wide variety of stages and both types of diabetes. In Type 1 diabetic patients with high normo- or microalbuminuria and with TNFR2 levels in the highest quartile, the cumulative incidence of reaching CKD stage 3 was extremely high (60\%) compared to those with the other lower 3 quartiles (less than $20 \%$ ) after 12 years of follow-up ${ }^{19}$. While type 2 diabetic patients with macroalbuminuria and with TNFR1 levels in the highest quartile had a nearly $80 \%$ cumulative incidence of progressing to ESRD after 12 years of follow-up, the rate was less than $20 \%$ in those with TNFR1 levels in the other lower 3 quartiles ${ }^{20)}$. Similar results were observed in each study when TNFR1 was used instead of TNFR2, but the TNFR2 levels were a bit more predictive in patients with T1D, and TNFR1 levels in patients with T2D. Also the results did not change after an adjustment for 
Table-1 The cumulative risk of CKD 3 or ESRD in diabetic patients of Joslin Clinic cohort according to the levels of circulating TNFR

\begin{tabular}{|c|c|c|c|c|c|c|c|}
\hline \multirow{2}{*}{$\begin{array}{l}\text { Study and } \\
\text { reference }\end{array}$} & \multirow{2}{*}{$\mathrm{N}$} & \multirow{2}{*}{ Type of diabetes } & \multirow{2}{*}{$\begin{array}{l}\text { Severity of } \\
\text { albuminuria }\end{array}$} & \multirow{2}{*}{ Outcome } & \multicolumn{2}{|c|}{$\begin{array}{c}\text { Cumulative } \\
\text { incidence in } \\
\text { patients who had }\end{array}$} & \multirow{2}{*}{$\begin{array}{l}\text { Follow-up } \\
\text { (years) }\end{array}$} \\
\hline & & & & & $\begin{array}{c}\text { Q4 } \\
\text { of } \\
\text { TNFR* }^{*}\end{array}$ & $\begin{array}{l}\text { Q1-Q3 } \\
\text { of } \\
\text { TNFR* }\end{array}$ & \\
\hline $\begin{array}{l}\text { 1st and 2nd Joslin } \\
\text { Kidney Study } 19 \text { ) }\end{array}$ & 628 & 1 & $\begin{array}{l}\text { High } \\
\text { normo- and } \\
\text { micro- }^{-}\end{array}$ & CKD 3 & 60 & $<20$ & 5 to 12 \\
\hline $\begin{array}{l}\text { Joslin Study of } \\
\text { the Genetics of } \\
\text { Kidney Disease } \\
\text { in T1D }\end{array}$ & 349 & 1 & Macro- $^{-}$ & ESRD & 90 & $<35$ & 5 to 18 \\
\hline $\begin{array}{l}\text { Joslin Study of } \\
\text { the Genetics of } \\
\text { Type } 2 \text { Diabetes }\end{array}$ & 330 & 2 & $\begin{array}{l}\text { Normo- } \\
\text { and micro- }\end{array}$ & ESRD & 25 & $<5$ & 8 to 12 \\
\hline $\begin{array}{l}\text { and Kidney } \\
\text { Complications }\end{array}$ & 80 & 2 & Macro- $^{-}$ & ESRD & 80 & $<20$ & 8 to 12 \\
\hline
\end{tabular}

${ }^{*}$ Round number

Q1-Q4, quartiles 1-4. Quartile boundaries for 25th, 50th, and 75th percentiles, respectively

other baseline markers, such as $\mathrm{HbAlc}$, albuminuria, and estimated GFR, in multivariate analysis. Meanwhile, ESRD was not associated with other inflammatory biomarkers, such as intercellular adhesion molecule 1 (ICAM-1), vascular cell adhesion molecule 1 (VCAM-1), interleukin 6 (IL-6), and $\mathrm{C}$ reactive protein $(\mathrm{CRP})$. Moreover, high concentrations of TNFRs also predicted cardiovascular and all-cause mortality, but these effects were smaller than those observed in ESRD. Recently, we observed similar results using a cohort of type 1 diabetic patients with macroalbuminuria. The cumulative incidence of reaching ESRD in patients with TNFR2 levels in the highest quartile was a remarkable ( $88 \%)$, compared to those in the other lower 3 quartiles (14-35\%) after 12 years of follow-up ${ }^{21)}$. Thus, the concentrations of TNFRs strongly predict early and late renal function loss in both types of diabetes, independent of classical risk factors such as GFR and albuminuria. The summary of the results obtained in the above studies is shown in Table-1.

\section{Understanding the pathogenesis of diabetic nephro- pathy: spontaneous type 2 diabetic mouse model}

\section{Characteristic and genetic background of the KK-A ${ }^{y}$ mouse}

Up to the present date, although many different varieties of diabetic mouse models are present, there is no mouse model that declines in renal function loss and subsequently reaches ESRD, even though an appropriate animal model is imperative to determine the etiology and better treatment of DN. We have especially focused on the KK and $\mathrm{KK}-\mathrm{A}^{\mathrm{y}}$ mouse because of close resemblances to human DN in terms of pathological findings and phenotypes such as hyperglycemia and an increase in albuminuria ${ }^{22}$. The KK mouse was established from a Japanese native mouse as an inbred mouse by Kondo $e t a l .{ }^{23)}$ in 1957 . Because the phenotypic characterizations, including hyperglycemia, obesity, and albuminuria, in this mouse are not marked, the $\mathrm{KK}-\mathrm{A}^{\mathrm{y}}$ mouse was established by transferring the $\mathrm{A}^{\mathrm{y}}$ allele at the agouti locus (yellow gene) into the KK mouse. Not surprisingly, the diabetic phenotypes in $\mathrm{KK}-\mathrm{A}^{\mathrm{y}}$ mice were more severe than those in KK mice.

At first, we therefore examined detailed renal lesions and the genetic background of $\mathrm{KK}-\mathrm{A}^{\mathrm{y}}$ mice. The pathological changes of the glomeruli, such as diffuse mesangial expansion with mesangial cell proliferation, segmental sclerosis, glomerular basement thickness, and podcyte loss, were consistent with those in the early stage of $\mathrm{DN}$, and were more severe than those in the KK mouse, as expected ${ }^{22)}$. Using a quantitative trait locus (QTL) analysis, Aoki et $a l .{ }^{24)}$ identified candidate loci for albuminuria in $270\left(\mathrm{KK}-\mathrm{A}^{\mathrm{y}} \times \mathrm{BALB} / \mathrm{c}\right) \mathrm{F} 2$ intercross mice. Gene loci contributing to albuminuria indicated a 
significant linkage to chromosome 9 (LOD: 3.8 ) in mice at 20 weeks. Interestingly, this locus was different from the loci of other diabetic-related phenotype, such as HbAlc and body weight, suggesting that the phenotypes of diabetes and nephropathy are controlled according to the diverse responsible gene.

\section{Therapeutic target for diabetic nephropathy: RAS inhibitor}

\section{1) Dual blockade of RAS}

Increased renal activation of RAS plays an important role in the pathogenesis of DN. A growing body of evidence is accumulating that the blockade of this system is a potent treatment strategy of DN. However, the efficacy of combination therapy with classical RAS inhibitors (ACE inhibitor and $\mathrm{ARB}$ ) has yet to be fully determined in patients with $\mathrm{DN}$, although a recent large scale clinical trial, the Veterans Affairs Nephropathy in Diabetes (VA NEPHRON-D) Study, which comprised of 1,448 patients with T2D and a wide range of GFR was stopped due to increased acute kidney injury (AKI) and hyperkalemia events ${ }^{25}$. Yamazaki et $a l .{ }^{26)}$ investigated whether the dual blockade of RAS is superior to either one of the two for the prevention of progressive $\mathrm{DN}$ in $\mathrm{KK}-\mathrm{A}^{\mathrm{y}}$ mouse. Enalapril (an ACE inhibitor) and/or losartan (an $\mathrm{ARB}$ ), especially in combination therapy, improved the level of albuminuria, and also inhibited the accumulation of carboxymethyllysine (CML), malondialdehyde (MDA), and 4-hydroxy-2-nonenal (HNE) in the kidney through tissue-specific activation of phospho-adenosine monophosphate activated protein kinase ( $\mathrm{p}$-AMPK) and adiponectin. These beneficial effects of RAS inhibitors were independent of a decrease in blood pressure. However, we do not know whether dual RAS blockade therapy prevents GFR loss because there is no diabetic mice model that progresses to ESRD.

2) 1, 25-dyhydroxyvitamin $\mathrm{D}_{3}\left[1,25(\mathrm{OH})_{2} \mathrm{D}_{3}\right]$ replacement therapy

As mentioned before, RAS inhibitors are an established therapy in patients with DN. However, these therapies suffer from the disadvantage of a compensatory increase in plasma renin activity due to their negative feedback system. Resnick $e t a l .{ }^{27)}$ reported that plasma levels of $1,25(\mathrm{OH})_{2} \mathrm{D}_{3}$ are inversely associated with blood pressure and plasma renin activity in normal and hypertensive subjects. Since then, mounting evidence has been accumulating that vitamin $\mathrm{D}$ receptor activation has a potent inhibitory effect on RAS. Therefore, a combination therapy with RAS inhibitors and a vitamin $\mathrm{D}$ analogue is presumed to be more effective than either one individually. Ohara et al. ${ }^{28)}$ examined the preventive effects of a combination therapy with $\mathrm{ARB}$ and $1,25(\mathrm{OH})_{2} \mathrm{D}_{3}$ in $\mathrm{KK}-\mathrm{A}^{\mathrm{y}}$ mouse. Not surprisingly, renal renin expression levels in mice treated with ARB significantly increased compared with control mice, but those levels were significantly decreased after combination therapy. The levels of albuminuria in mice with the combination therapy were significantly lower than those in mice with ARB monotherapy. These beneficial effects might be related to suppressed renal expressions of extracellular signal regulated kinase 1 and 2 (ERK1/2) - transforming Growth Factor- $\beta 1$ (TGF $\beta 1$ ) pathway in addition to renin activity. Recently, the Selective Vitamin D Receptor Activator for Albuminuria Lowering (VITAL) Study showed that another vitamin D analogue, paricalcitol, in addition to an ACE inhibitor or ARB therapy, reduced residual albuminuria in a dose dependent manner in patients with T2D and nephropathy ${ }^{29)}$. Although, whether paricalcitol postpones the progression to ESRD was not determined, this approach might be a novel strategy to lowering the residual renal risk in patients with diabetes.

3) Direct renin inhibitor: aliskiren

In contrast to classical RAS blockers, aliskiren blocks RAS by directly inhibiting plasma renin activity and preventing the formation of both angiotensin (Ang) I and Ang II. The Aliskiren in the Evaluation of Proteinuria in Diabetes (AVOID) trial demonstrated that the addition of aliskiren to ARB provides an additive antiproteinuric effect compared to ARB alone ${ }^{30}$. Whereas, the Aliskiren Trial in Type 2 Diabetes Using Cardio-Renal Endpoints (ALTITUDE) study concluded that the dual blockade of the RAS with aliskiren should no longer be used in patients with T2D and chronic kidney disease (CKD) due to a lack of a clinical benefit on cardiorenal end points, although both trials showed a certain level of reduction in albuminuria ${ }^{31)}$. To understand the mechanism of action of aliskiren in the prevention of renal disease 
progression, Furukawa et al. ${ }^{32}$ examined the renoprotective effects of aliskiren in the $\mathrm{KK}-\mathrm{A}^{\mathrm{y}}$ mouse. Aliskiren dramatically ameliorated the albuminuria and renal fibrosis by improving renal inflammation and the alteration of matrix metalloproteinase (MMP) and/or tissue inhibitor of metalloproteinase (TIMP) expression through the activation of mitogen-activated protein kinase (MAPK) and nuclear factor-kappa $\mathrm{B}(\mathrm{NF}-\kappa \mathrm{B})$. The results demonstrated that aliskiren monotherapy might have beneficial effects in an early stage of DN.

4) Approaches in the use of RAS inhibitors in patients with diabetes

Classical RAS inhibitors (ACE inhibitor and ARB) are a first-line treatment for diabetic patients with hypertension, irrespective of the degree of albuminuria. If diabetic patients have microalbuminuria but not hypertension, we should consider the administration of a RAS inhibitor. There is a clear indication for the use of RAS inhibitors in diabetic patients with macroalbuminuria to prevent progressive GFR loss, even if patients are normotensive. There is no clear indication for the use of RAS inhibitors in diabetic patients with normoalbuminuria and normotension. The flowchart for the use of RAS inhibitors is shown in Figure-3.

At this moment, a dual classical RAS blockade therapy should be performed with careful attention to AKI and hyperkalemia in patients with diabetes. The ongoing (to be completed in December 2015) Preventing ESRD in Overt Nephropathy of Type 2 Diabetes (VALID) study, which comprises of 100 patients with T2D and advanced renal function (serum creatinine levels and albuminuria are $1.8-3.5 \mathrm{mg} / \mathrm{d} l$ and more than $1 \mathrm{~g} / \mathrm{g} \mathrm{Cr}$ ), will be used to elucidate the efficacy of dual RAS blockade therapy for prevention of ESRD ${ }^{33)}$. This study may be able to make a final decision about the significance of a dual RAS blockade therapy in patients with DN.

\section{Conclusions}

Over the past 2 decades, significant progress has been made in treatments that slow the progression of or prevent DN. Multifactorial therapies, including strict blood glucose control, blood pressure control using RAS inhibitors, lipid management, and so on, have achieved great success in terms of the reduction of albuminuria and cardiovascular events.

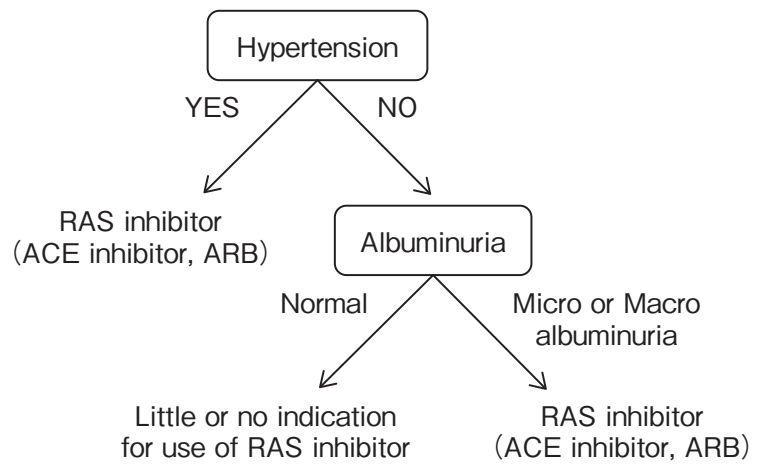

Figure-3 Approach in the use of RAS inhibitors in patients with diabetes

If diabetic patients have hypertension, the use of RAS inhibitors is highly recommended. In patients without hypertension, the indication of RAS inhibitors varies according to the level of albuminuria. If diabetic patients have micro- or macro-albuminuria, but not normoalbuminuria, there is a clear indication for the use of RAS inhibitor.

However, the leading cause of ESRD is still diabetes at this moment worldwide. More clinical research into finding novel biomarkers to predict future GFR loss, and also basic research into finding target molecules to prevent progressive $\mathrm{DN}$, is needed to neutralize this devastating disease completely.

\section{Acknowledgements}

We would like to offer our special thanks to Dr. Andrzej Krolewski (Joslin Diabetes Center, MA) and also all our colleagues of the Diabetic Nephropathy Research Group.

\section{Financial disclosure}

The authors declare no competing interests.

\section{Funding/Support}

None declared.

\section{References}

1) The National Health and Nutrition Survey 2012: Accessed at http://www.mhlw.go.jp/stf/houdou/0000032074. html on 1st May 2014.

2) Kadowaki T: 108th Scientific Meeting of the Japanese Society of Internal Medicine: invited lecture: 4. Molecular mechanism and treatment strategy of type 2 diabetes. Nihon Naika Gakkai Zasshi 2011; 100: 24372446.

3) Japanese Society for Dialysis Therapy: The Current 
State of Chronic Dialysis Treatment in Japan (as of December 31, 2012). Accessed at http://www.jsdt.or.jp/ overview_confirm.html on 1st May 2013.

4) The effect of intensive treatment of diabetes on the development and progression of long-term complications in insulin-dependent diabetes mellitus. The Diabetes Control and Complications Trial Research Group. N Engl J Med, 1993; 329: 977-986.

5) Lewis EJ, Hunsicker LG, Bain RP, et al: The effect of angiotensin-converting-enzyme inhibition on diabetic nephropathy. The Collaborative Study Group. N Engl J Med, 1993; 329: 1456-1462.

6) Brenner BM, Cooper ME, de Zeeuw D, et al: Effects of losartan on renal and cardiovascular outcomes in patients with type 2 diabetes and nephropathy. N Engl J Med, 2001; 345: 861-869.

7) de Boer IH, Rue TC, Hall YN, et al: Temporal trends in the prevalence of diabetic kidney disease in the United States. JAMA, 2011; 305: 2532-2539.

8) Fioretto P, Steffes MW, Sutherland DE, et al: Reversal of lesions of diabetic nephropathy after pancreas transplantation. N Engl J Med, 1998; 339: 69-75.

9) Perkins BA, Ficociello LH, Silva KH, et al: Regression of microalbuminuria in type 1 diabetes. N Engl J Med, 2003; 348: 2285-2293.

10) Hovind P, Rossing P, Tarnow L, et al: Remission and regression in the nephropathy of type 1 diabetes when blood pressure is controlled aggressively. Kidney Int, 2001; 60: 277-283.

11) Hovind $\mathrm{P}$, Rossing $\mathrm{P}$, Tarnow $\mathrm{L}$, et al: Remission of nephrotic-range albuminuria in type 1 diabetic patients. Diabetes Care, 2001; 24: 1972-1977.

12) Rossing K, Christensen PK, Hovind P, et al: Remission of nephrotic-range albuminuria reduces risk of end-stage renal disease and improves survival in type 2 diabetic patients. Diabetologia 2005; 48: 2241-2247.

13) Skupien J, Warram JH, Smiles AM, et al: The early decline in renal function in patients with type 1 diabetes and proteinuria predicts the risk of end-stage renal disease. Kidney Int, 2012; 82: 589-597.

14) Krolewski AS, Niewczas MA, Skupien J, et al: Early progressive renal decline precedes the onset of microalbuminuria and its progression to macroalbuminuria. Diabetes Care, 2014; 37: 226-234.

15) MacIsaac RJ, Tsalamandris $C$, Panagiotopoulos $S$, et al: Nonalbuminuric renal insufficiency in type 2 diabetes. Diabetes Care, 2004; 27: 195-200.

16) Caramori ML, Fioretto P, Mauer M: Low glomerular filtration rate in normoalbuminuric type 1 diabetic patients: an indicator of more advanced glomerular lesions. Diabetes, 2003; 52: 1036-1040.

17) Yokoyama $\mathrm{H}$, Sone $\mathrm{H}$, Oishi $\mathrm{M}$, et al: Prevalence of albuminuria and renal insufficiency and associated clinical factors in type 2 diabetes: the Japan Diabetes Clinical Data Management study (JDDM15). Nephrol Dial Transplant, 2009; 24: 1212-1219.

18) Ninomiya $T$, Perkovic $V$, de Galan BE, et al: Albuminuria and kidney function independently predict cardiovascu- lar and renal outcomes in diabetes. J Am Soc Nephrol, 2009; 20: 1813-1821.

19) Gohda T, Niewczas MA, Ficociello LH, et al: Circulating TNF receptors 1 and 2 predict stage 3 CKD in type 1 diabetes. J Am Soc Nephrol, 2012; 23: 516-524.

20) Niewczas MA, Gohda T, Skupien J, et al: Circulating TNF receptors 1 and 2 predict ESRD in type 2 diabetes. J Am Soc Nephrol, 2012; 23: 507-515.

21) Skupien J, Warram J, Niewczas M, et al: Synergism between circulating tumor necrosis factor receptor 2 and $\mathrm{HbAlc}$ in determining renal decline during 5-18 years of follow-up in patients with type 1 diabetes and proteinuria. Diabetes Care, 2014; 37: 2601-2608.

22) Ito T, Tanimoto M, Yamada K, et al: Glomerular changes in the $\mathrm{KK}-\mathrm{Ay} / \mathrm{Ta}$ mouse: a possible model for human type 2 diabetic nephropathy. Nephrology (Carlton), 2006; 11: 29-35.

23) Kondo K, Nozawa K, Tomita T, et al: Inbred strains resulting from Japanese mice. Bull Exp Anim, 1967; 6: $107-112$.

24) Aoki T, Kaneko S, Tanimoto M, et al: Identification of quantitative trait loci for diabetic nephropathy in KK-Ay/Ta mice. J Nephrol, 2012; 25: 127-136.

25) Fried LF, Emanuele N, Zhang JH, et al: Combined angiotensin inhibition for the treatment of diabetic nephropathy. N Engl J Med, 2013; 369: 1892-1903.

26) Yamazaki T, Tanimoto M, Gohda T, et al: Combination effects of enalapril and losartan on lipid peroxidation in the kidneys of KK-Ay/Ta mice. Nephron Exp Nephrol, 2009; 113: e66-e76.

27) Resnick LM, Muller FB, Laragh JH: Calcium-regulating hormones in essential hypertension. Relation to plasma renin activity and sodium metabolism. Ann Intern Med, 1986; 105: 649-654.

28) Ohara I, Tanimoto M, Gohda T, et al: Effect of combination therapy with angiotensin receptor blocker and 1,25-dihydroxyvitamin D (3) in type 2 diabetic nephropathy in KK-A (y)/Ta mice. Nephron Exp Nephrol, 2011; 117: e124-e132.

29) de Zeeuw D, Agarwal R, Amdahl M, et al: Selective vitamin $\mathrm{D}$ receptor activation with paricalcitol for reduction of albuminuria in patients with type 2 diabetes (VITAL study) : a randomised controlled trial. Lancet, 2010; 376: 1543-1551.

30) Parving HH, Persson F, Lewis JB, et al: Aliskiren combined with losartan in type 2 diabetes and nephropathy. N Engl J Med, 2008; 358: 2433-2446.

31) Parving HH, Brenner BM, McMurray JJ, et al: Cardiorenal end points in a trial of aliskiren for type 2 diabetes. N Engl J Med, 2012; 367: 2204-2213.

32) Furukawa M, Gohda T, Tanimoto M, et al: Pathogenesis and novel treatment from the mouse model of type 2 diabetic nephropathy. ScientificWorldJournal, 2013; 2013: 928197.

33) Remuzzi G: Preventing ESRD in Overt Nephropathy of Type 2 Diabetes (VALID). Accessed at http://clinicalt rials.gov/show/NCT00494715 on 1st May 2014. 